\title{
Lymphoedema Presenting with Pleural Effusion: A Case Report
}

\author{
Minhaj Rahim Choudhury', M. Masudul Hassan², M. Mustafizur Rahman Ratan³, M. Mizanur Rahman Bhuyia², \\ Farzana Kabir ${ }^{4}$, M. Safiuddin ${ }^{5}$, Ferdous Hakim ${ }^{6}$
}

${ }^{1}$ Associate Professor. ${ }^{2}$ Medical Officer, ${ }^{4}$ FCPS Student, Department of Medicine, ${ }^{3}$ Associate Professor, Department Of Cardiothoracic Surgery, ${ }^{5}$ Associate. Prof. of Cardiology, BSMMU, Shahbag, Dhaka. ${ }^{6}$ Resident Medical Officer, Cardiology, Square Hospitals Ltd., Panthapath, Dhaka.

\begin{abstract}
:
Lymphatic obstruction either congenitally determined (primary) or by a consequence of acquired lymphatic failure because of obstruction or damaged lymphatics (secondary). Milroy disease is an example of primary lymphoedema where edema is present at birth or soon thereafter. A 16 year old young boy presented with bilateral leg with scrotal oedema since birth with ups and downs of his symptoms. According to the statement of the mother the boy was seen by several physicians and also attended several tertiary hospitals in Dhaka city and eventually was diagnosed as a case of Milroy disease in 2003. Patient was in a reasonably stable condition till July 2008 when he developed massive pleural effusion on right side and chylous fluid was aspirated. After that he developed five episodes of right sided pleural effusion at one to three month interval. Thoracic surgeon was consulted and intra thoracic tube drainage and pleurodesis was done on 19. 02. 2009. After couple of weeks repeat chest $\mathrm{x}$-ray revealed left sided pleural effusion and again left sided pleurodesis was performed. He is now clinically stable except mild oedema in his both feet.
\end{abstract}

[BSMMU J 2010; 3(1): 40-43]

\section{Introduction:}

Lymphoedema (LE) is the abnormal buildup of fluid in an arm or leg due to a malfunction or malformation in the lymphatic system ${ }^{1}$. In long-lasting LE, interstitial accumulation of proteins ${ }^{2}$, inflammatory cells, adipose tissue hypertrophy and fibrosis make pitting less evident. ${ }^{3}$ Erysipelas (cellulitis) is common. It can be congenitally determined (primary) or by a consequence of acquired lymphatic failure because of obstruction or damaged lymphatic's (secondary) ${ }^{4,5}$. Primary LE is traditionally classified according to the time of onset: congenital LE, LE praecox or LE tarda. Congenital LE is present at birth or soon thereafter. LE may be the only clinical sign (Milroy disease). LE praecox presents between 1 and 35 years of age and is mostly sporadic. Familial cases of LE praecox are called the Meige syndrome. In children, nearly all LEs are classified as primary with an estimated prevalence of $1.15 / 100.000$ persons $<20$ years old ${ }^{6}$. Genetic conditions associated with LE from solitary case reports estimates of approximating $1 / 500$ birth in case of Klinefelter syndrome. ${ }^{7}$ A study by Dale ${ }^{8}$ of 312 index patients younger than 36 years with primary LE reported a frequency of $1 / 6000$ at birth and a sex ratio of one male to three females. In a large study from Smeltzer et al. ${ }^{6}$ analyzing 291 children, only 42

Address for Correspondence: Dr. Minhaj Rahim Choudhury, Associate Professor, Department of Medicine, BSMMU, Dhaka
(14\%) had a family history of LE. Infectious LE (filiariasis) is most frequent in tropical regions. In endemic regions, the prevalence in children aged $0-5$ years is $18.9 \%{ }^{9}$.

Persistent, painless swelling, typically of one (or both) lower limb(s), is usually the first sign of LE in Milroy's disease. Swelling is often present at birth or soon afterwards. Due to the accumulation of protein-rich interstitial fluid, thickening of the skin over the digits occurs. When there is an inability to pinch up a fold of skin between the second and third toe on the dorsum of the foot is called a positive Stemmer sign. This sign is a sensitive test for LE that does not give false positive findings. ${ }^{10,17}$ In a study of 71 patients from 10 families with Milroy disease had VEGFR3 mutations. ${ }^{23}$ Brice et al described an onset of LE at birth in $97 \%$ of the patients; distal oedema (94\%), bilateral (85\%), unilateral (14\%), hydrocele in males (37\%), prominent veins (23\%), cellulitis (20\%) and ‘ski jump' nails(14\%). Visceral forms of lymphatic impairment can accompany LE sometimes. If lymphostasis occurs in the cisterna chyli, the drainage from the entire intestine can be deficient. Primary chylous ascites is closely correlated to generalized lymphatic dysplasia. Chyle can also occur in the thorax (chylothorax), urine (chyluria) or can leak into the gut (protein-losing enteropathy) ${ }^{12}$. Lymphangiography is in general obsolete to diagnose LE, but is only useful to visualize ectasia via injection in a cutaneous vesicle ${ }^{13}$. MRI and videocapsule endoscopy may confirm the diagnosis in visceral form. ${ }^{14,15}$ 


\section{Case report:}

Md. Humayun Kabir, a 16 year old pleasant boy, hailing from Nawabgonj, Dhaka presented with swelling of both lower limbs and scrotum since birth with an exacerbation and remission of symptoms. His mother stated that she noticed scrotal swelling and mild leg oedema at birth. Since then several physicians were consulted and was treated with several drugs which she could not specify except diuretics. He also tried supportive measures like elevation of legs at bedtime, crepe bandage, massage etc; however, swelling did not subside rather progressed gradually.

In 1995, the boy was suspected as a case of lymphoedema in a tertiary hospital OPD and treated conservatively with pressure bandage/compression stockings but oedema persisted with ups and downs. In 2003, he got himself admitted in a tertiary care hospital for respiratory tract infection and was diagnosed as a case of Milroy's disease. Respiratory infection was improved with antibiotic. In the same year he was seen by a vascular surgeon and underwent lymphoscientigraphy which revealed reduced lymph flow in both lower limbs, more on the left side with patent thoracic duct. Patient was in a stable condition till July 2008, when he developed shortness of breath, and was found to have massive pleural effusion in the right side, which was milky white in color (chylous effusion). He had 5 episodes of shortness of breath at 1 to 3 month interval. Diagnostic and therapeutic pleurocentesis was done and in each episode revealed similar type of chylous effusion. History, clinical examination \& investigations excluded tuberculosis, filariasis, chronic cellulitis, heart failure, liver disease and lymphoma .Patient was presented in our departmental grand round. Thoracic surgeon was consulted and intra-thoracic tube was placed with an aim to pleurodesis (Fig.-1). Following the procedure on right side, his dyspnoea and features of pleural effusion

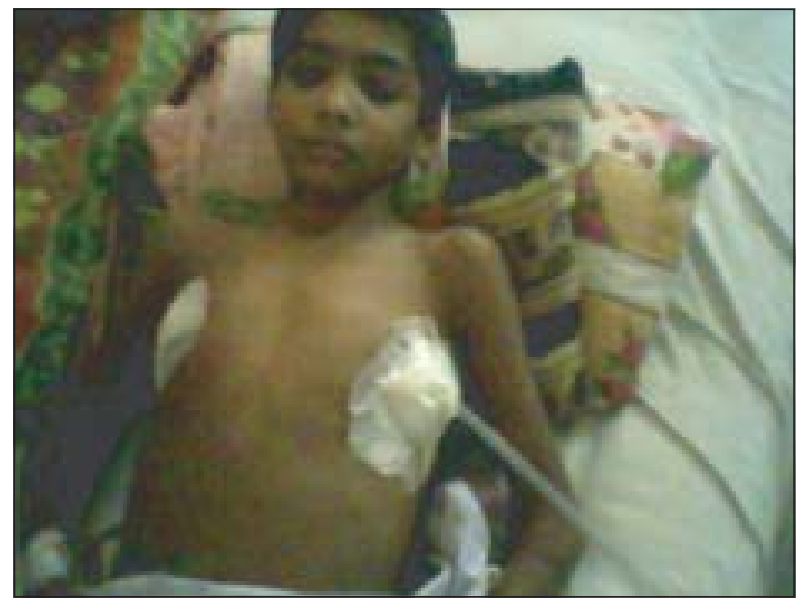

Fig.-1: Photograph showing the intrathoracic tube drainage subsided then after couple of weeks he developed left sided pleural effusion and again pleurodesis was done on that side.

Complete blood count, haemoglobin level, erythrocyte sedimentation rate, urine routine examinations, serum albumin, SGPT, serum TSH were normal on several occasion during the course of illness. ECG \& Echo were also normal. Mantouex test, ICT for Filaria were also negative. X-ray showed massive pleural effusion on right side. Pleural fluid study on different occasions showed exudative fluid rich in triglyceride. Gram stain and culture were negative, AFB was also negative, color was milky in every occasion, pleural biopsy finding was nonspecific.

Duplex ultrasound of lower limbs showed patent blood vessels. Lymphoscintigraphy was done on 01.06.2003. Abnormal lymphatic flow was in left lower limb, only the right inguinal lymph node was visualized. Thoracic duct was patent. On 30.07.08 lymphoscientigraphy was repeated and bilateral complete obstruction of lymphatic flow was observed. Therefore lymphatic flow in the thoracic duct was not visualized.

Pleurodesis, on right side was done on 19.02.2009 with tetracycline. After couple of weeks a repeat chest x-ray revealed left sided pleural effusion and again pleurodesis was done with tetracycline on 05.03.2009 on left side. Repeat chest $x$-ray after three weeks was normal. He is now clinically stable except mild oedema in both lower limbs.

\section{Discussion:}

The patient had nonpitting oedema for 16 years. Nonpitting is characteristic of lymphoedema which involves extremity including digits. In secondary causes of lymphoedema, the swelling may first be apparent only in the proximal portion of the limb, or it may affect only a portion of the distal limb. Patient was evaluated for cellulitis and lymphangitis. There was no macular or maculopapular erythematous rash, or a red macular line or pain of lymphangitis. Heart failure, chronic liver disease, nephrotic syndrome, as a cause of oedema was ruled out. Precordium examination was normal, his liver was not enlarged. The primary forms of lymphoedema have a relative lack of fibrosis that permits greater amounts of oedema to form ${ }^{16}$. The boy had the swelling since birth but fibrosis was not prominent; the oedema was not so farm, which favor primary lymphoedema. Kaposi-Stemmer sign (fold of skin at the base of the second toe on the dorsal foot cannot be pinched) was positive consistent with lymphoedema ${ }^{10,17}$. Lymphangiosarcoma may occur with chronic lymphedema, usually in patients with massive and protracted oedema 
especially in postmastectomy, lymphoedematous arm (Stewart-Treves syndrome), and filarial infection ${ }^{18-22}$.

In July 2008, he presented with breathlessness and diagnosed as chylous effusion, which was progressive and bilateral. The patient admitted repeatedly in hospital for breathlessness and aspiration was done every time followed by relief of his symptom temporarily. Standard treatment for pleural effusion is aspiration of pleural fluid and treatment of the cause. Parapneumonic effusion was suspected and antibiotic given but effusion persisted and breathlessness was increasing. Then tuberculosis, lymphoma was excluded after clinical and laboratory evaluation. His lymphnodes were never palpable and he had no organomegaly. His Mantouex test was negative and ESR was also not raised. Pleural fluid examination for gram stain, $\mathrm{C} / \mathrm{S}$ and AFB was negative. Fluid was exudative and chylous (white and triglyceride rich).

Lymphoscientigraphy (radionuclide imaging) is usually preferred over lymphangiography ${ }^{23-25}$. MRI and CT scan may complement information obtained via lymphoscintigraphy ${ }^{26}$. In this patient lymphoscientigaphy was done which shows obstruction of lymphatic channel. Other routine and specific investigations were normal.

Patient was advised to avoid I.V. channels, tourniquet in affected limb. Meticulous skin hygiene and nail care was advised and advised to use skin moisturizer and topical antibiotic solutions after injury. These will help to control limb swelling since the underlying disease cannot usually be corrected $^{24,25,28-32}$. Furthermore, if left untreated, lymphoedema tends to gradually progress over time and to inhibit the activities of daily living ${ }^{16}$. No drugs have been shown to be beneficial and diuretic should not be used ${ }^{28}$, 30 . Treatment advised to the patient was exercise, gradient pressure garments, massage therapy. In some cases, external pneumatic compression may help ${ }^{24,25,31}$. Gradient pressure garments (lymphoedema sleeve or stocking) generate greater pressures distally than proximally, thereby promoting mobilization of the oedema fluid ${ }^{24,25}$. The bandage wraps, combined with manual massage, may change an initially resistant limb to one with less oedema ${ }^{32,33}$. Massage therapy or manual lymphoedema drainage (MLD) techniques use light pressure applied to the torso to help open collateral channels connecting the lymphoedematous area with major draining areas at the base of other limbs 24,32 , Our patient was educated and trained.

Diuretic and coumarin have been tried but it was not satisfactory. Our patient was given diuretic in the past but there was no improvement. Surgical method for treating lymphoedema consists of removing the subcutaneous fat and fibrous tissue. An alternative approach is lymphatic microsurgery. Gene therapy to develop new lymphangioles in the affected limbs is a potential clinical remedy in the future.

Progressive pleural effusion of this patient made the patient bedridden for breathlessness. Accumulation was due to obliteration of lymphatic channels which progress as a part of progression of Milroy's disease. Patient was dyspnoeic and was unable to sleep at all. Conventional palliative treatment failed to improve symptoms or to stop the progression. Diuretic and repeated aspiration failed and patient became breathless. For lymphatic obstruction, there is no curative treatment but pluerodesis may stop accumulation in pleural space, by eliminating potential space $^{34}$. In our patient, pluerodesis was performed by thoracic surgeon. Now the patient has no difficulties in respiration for last one year. Decompressing physiotherapy reduces leg oedema and pluerodesis may be life saving in patients with pleural effusion. Milroy's disease is uncommon and development of pleural effusion is extremely rare.

\section{Conclusion:}

Most Lymphoedema in children occur as primary Lymphoedema. If it starts since birth it is called Milroy's disease. For long standing oedema, treatment will focus on non-operative options, such as manual lymphedema drainage, compression therapy and exercises with the close involvement of the parents. Primary LE does not only involve limbs but may also involve viscera. In this case pleural effusion was due to lymphatic obstruction. It was recurrent and developed five episodes within one year. Pluerodesis was done and his respiratory symptoms subsided. As lymphoedema is a chronic disease, life-long follow up is useful for preventing complications and monitoring the effects of treatment.

\section{References:}

1. Mortimer PS, Levick JR. Chronic peripheral oedema: the critical role of the lymphatic system. Clin Med 2004; 4: 448-53.

2. Gaffney RM, Casley-Smith JR. Excess plasma proteins as a cause of chronic inflammation and lymphedema: biochemical estimations. J Pathol 1981; 133: 229-42.

3. Warren AG, Brorson H, Borud LJ, Slavin SA. Lymphedema: a comprehensive review. Ann Plast Surg 2007; 59: 464-72.

4. Belov ST. Anatomopathological classification of congenital vascular defects. Semin Vasc Surg 1993; 6: 219-24.

5. Belov ST. Classification of vascular defects. Int Angiol 1990; 9: 141-6 Belov ST. Classification of vascular defects. Int Angiol 1990; 9: 141-6. 
6. Smeltzer DM, Gunnar B, Schirger A. Primary lymphedema in children and adolescents: a follow-up study and review. Pediatrics 1985: 76: 206-18.

7. Greenlee R, Hoyme H, Witte M, Crowe P, Witte C. Developmental disorders of the lymphatic system. Lymphology 1993; 26: 156-8.

8. Dale RF. The inheritance of primary lymphedema. J Med Genet 1985; 22: 274-8.

9. Ramaiah KD, Das PK, Vanamail P, Pani SP. Impact of 10 years of diethylcarbamazine and ivermectin mass administration on infection and transmission of lymphatic filariasis. Trans R Soc Trop Med Hyg 2007; 101: 555-63.

10. Stemmer R. Stemmer's sign: possibilities and limits of clinical diagnosis of lymphedema. Wien Med Wochenschr 1999; 149: 85-6.

11. Brice G, Child AH, Evans A, et al. Milroy disease and the VEGFR-3 mutation phenotype. J Med Genet 2005; 42: 98102

12. Aalami OO, Allen DB, Organ CH. Chylous ascites: a collective review. Surgery 2000; 128: 761-78

13. Howarth D, Gloviczki P. Lymphoscintigraphy and lymphangiography of lymphangectasia. J Nucl Med 1998; 9: 683-90.

14. Chamouard P, Nehme-Schuster H, Simler J-M, Finck G, Baumann R, Pasquali J-L. Videocapsule endoscopy is useful for the diagnosis of intestinal lymphangiectasia. Digest Liver Dis (Official Journal of the Italian Society of Gastroenterology and the Italian Association for the Study of the Liver) 2006; 38: 699-703.

15. Campisi C, Bellini C, Errata C. Diagnosis and management of primary chylous ascites. J Vasc Surg 2006; 43: 1244-8.

16. Casley-Smith, JR. Alterations of untreated lymphedema and it's grades over time. Lymphology 1995; 28: 174.

17. Kerchner, K, Fleischer, A, Yosipovitch, G. Lower extremity lymphedema update: pathophysiology, diagnosis, and treatment guidelines. J Am Acad Dermatol 2008; 59: 324.

18. Woodward, AH, Ivins, JC, Soule, EH. Lymphangiosarcoma arising in chronic lymphedematous extremities. Cancer 1972; 30: 562 .

19. Stewart, FW, Treves, N. Classics in oncology: Lymphangiosarcoma in postmastectomy Iymphedema: A report of six cases in elephantiasis chirurgica. CA Cancer J Clin 1981; 31: 284.

20. Tomita, K, Yokogawa, A, Oda, Y, Terahata, S. Lymphangiosarcoma in postmastectomy lymphedema
(Stewart-Treves syndrome): ultrastructural and immunohistologic characteristics. J Surg Oncol 1988; 38:275.

21. Cozen W, Bernstein L, Wang F. The risk of angiosarcoma following primary breast cancer. Br J Cancer 1999; 81: 532.

22. Muller R, Hajdu SI, Brennan MF. Lymphangiosarcoma associated with chronic filarial lymphedema. Cancer 1987; 59: 179.

23. McNeill, GC, Witte, MH, Witte, CL. Whole-body lymphangioscintigraphy: preferred method for initial assessment of the peripheral lymphatic system. Radiology 1989; 172:495.

24. The diagnosis and treatment of peripheral lymphedema. Consensus document of the International Society of Lymphology Executive Committee. Lymphology 1995; 28: 113.

25. Rockson SG, Miller LT, Senie R, et al. American Cancer Society Lymphedema Workshop. Workgroup III: Diagnosis and management of lymphedema. Cancer 1998; 83: 2882.

26. Rockson SG. Lymphedema. Am J Med 2001; 110: 288.

27. Ganz PA. The quality of life after breast cancer-solving the problem of lymphedema [editorial; comment]. N Engl J Med 1999; 340: 383.

28. Brennan, MJ. Lymphedema following the surgical treatment of breast cancer: A review of pathophysiology and treatment. J Pain Symptom Manage 1992; 7: 110.

29. Campisi C. A rational approach to the management of lymphedema. Lymphology 1991; 24: 48.

30. Tunkel RS, Lachmann E. Lymphedema of the limb. An overview of treatment options. Postgrad Med 1998; 104: 131.

31. Brennan MJ, Miller LT. Overview of treatment options and review of the current role and use of compression garments, intermittent pumps, and exercise in the management of lymphedema. Cancer 1998; 83: 2821.

32. Zanolla R, Monzeglio C, Balzarini A, Martino G. Evaluation of the results of three different methods of postmastectomy lymphedema treatment. J Surg Oncol 1984; 26: 210.

33. Badger CM, Peacock JL, Mortimer PS. A randomized, controlled, parallel-group clinical trial comparing multilayer bandaging followed by hosiery versus hosiery alone in the treatment of patients with lymphedema of the limb. Cancer 2000; 88: 2832.

34. Sivaraja SB, Sunder V, Vinoth Kumar V, Ranganathan. D, An Interesting Case of a British Orphan Lung Disease (BOLD), Pulmon 2007; 9: 24: 69 - 72. 